\title{
多重响应性超支化星形聚合物的合成与组装以及控制释放研究
}

\author{
张文建范溦李敏洪春雁* 潘才元 \\ (中国科学院软物质化学重点实验室 中国科学技术大学 高分子科学与工程系 合肥 230026)
}

\begin{abstract}
摘要 含二硫键的自引发单体与 2-(2-甲氧基乙氧基)乙基甲基丙烯酸酯( $\mathrm{MEO}_{2} \mathrm{MA}$ )进行自缩合乙烯基共聚合得到超支 化 $\mathrm{PMEO}_{2} \mathrm{MA}\left(\mathrm{H}-\mathrm{PMEO}_{2} \mathrm{MA}\right)$. 以它作大分子引发剂, 引发二甲氨基乙基甲基丙烯酸酯(DMAEMA)进行原子转移自由 基聚合, 合成了具有温度、 $\mathrm{pH}$ 以及氧化还原多重响应性的超支化星形聚合物 H-PMEO ${ }_{2}$ MA-star-PDMAEMA. 证明了 $\mathrm{H}_{-} \mathrm{PMEO}_{2} \mathrm{MA}$ 有低临界溶液温度(LCST); 研究了 PDMAEMA 链段的长度和溶液的 $\mathrm{pH}$ 值对超支化星形聚合物的 LCST 的影响. 当 $\mathrm{H}-\mathrm{PMEO}_{2} \mathrm{MA}$-star-PDMAEMA 水溶液温度从 $2{ }^{\circ} \mathrm{C}$ 升高至室温, $\mathrm{H}-\mathrm{PMEO}_{2} \mathrm{MA}$ 变成疏水性而发生聚集, 形成 以 $\mathrm{H}-\mathrm{PMEO}_{2} \mathrm{MA}$ 为核, PDMAEMA 为壳的胶束. 在胶束形成过程中, 将尼罗红装载到这种聚合物胶束中, 形成释药系 统, 研究了 $\mathrm{pH}$ 、氧化还原响应性释药性能.

关键词 自缩合乙烯基聚合; 原子转移自由基聚合; 超支化星形聚合物; 多重响应性; 控制释放
\end{abstract}

\section{Multi-responsive Hyperbranched Star Copolymer: Synthesis, Self-assembly and Controlled Release}

\author{
Zhang, Wenjian Fan, Wei Li, Min Hong, Chunyan* Pan, Caiyuan \\ (CAS Key Laboratory of Soft Matter Chemistry, Department of Polymer Science and Engineering, University of Science and \\ Technology of China, Hefei 230026)
}

\begin{abstract}
Multi-responsive (temperature, $\mathrm{pH}$ and redox) hyperbranched star polymers, poly(2-(2-methoxyethoxy)ethyl methacrylate)-star-poly(dimethylaminoethyl methacrylate) ( $\mathrm{H}-\mathrm{PMEO}_{2} \mathrm{MA}$-star-PDMAEMA) have been successfully synthesized by self-condensing vinyl polymerization of disulfide-based inimer and $\mathrm{MEO}_{2} \mathrm{MA}$ first, and subsequently atom transfer radical polymerization of DMAEMA with $\mathrm{H}-\mathrm{PMEO}_{2} \mathrm{MA}$ as macroinitiator. The $M_{\mathrm{n}}$ and $M_{\mathrm{w}} / M_{\mathrm{n}}$ of the H-PMEO $\mathrm{MA}_{2}$ were $8300 \mathrm{~g} / \mathrm{mol}$ and 2.61, respectively. $\mathrm{H}-\mathrm{PMEO}_{2} \mathrm{MA}$-star-PDMAEMAs with different molecular weights were obtained by adjusting the polymerization time. The molecular weight of the hyperbranched star copolymer increased but the polydispersity index (PDI) decreased with increasing polymerization time. Since the PDI of the PDMAEMA formed by ATRP is low, with the molecular weight increase of the PDMAEMA, the relative amount of $\mathrm{H}_{-} \mathrm{PMEO}_{2} \mathrm{MA}$ in the hyperbranched star copolymers becomes less; as a result, the influence of the core $\mathrm{H}-\mathrm{PMEO}_{2} \mathrm{MA}$ 's PDI on the hyperbranched star copolymers decreases. UV/Vis TU-1901 spectrophotometer was used to investigate the lower critical solution temperature (LCST) of the resultant polymer. The LCST of $\mathrm{H}-\mathrm{PMEO}_{2} \mathrm{MA}$ is relatively low $\left(2-10{ }^{\circ} \mathrm{C}\right)$. The effects of the compositions and $\mathrm{pH}$ of the solution on LCST of the hyperbranched star copolymers were studied. The LCST increased with the chain length increase of PDMAEMA. The $\mathrm{pH}$ of the solution has a significant impact on the LCST of the hyperbranched star copolymers. With decrease of the $\mathrm{pH}$ value, the protonation degree of PDMAEMA increased, the repulsion between the chain segments enhanced, making the aggregation of the H-PMEO ${ }_{2} \mathrm{MA}$-star-PDMAEMA molecules become difficult, and as a result, the water-solubility of the hyperbranched star copolymers enhanced. In addition, when temperature of the aqueous solution raised from $2{ }^{\circ} \mathrm{C}$ to room temperature, the spherical micelles with $\mathrm{H}-\mathrm{PMEO}_{2} \mathrm{MA}$ as core and PDMAEMA as shell were formed. During the formation of spherical micelles in the aqueous solution of $\mathrm{H}_{-} \mathrm{PMEO}_{2} \mathrm{MA}$-star-PDMAEMA and Nile Red, the Nile Red was successfully encapsulated in the micelles. The controlled release of this system, in which Nile Red was used as model drug, was investigated, the results showed that this system is $\mathrm{pH}$ and redox-responsive, and may have potential application in drug delivery.

Keywords self-condensing vinyl polymerization (SCVP); atom transfer radical polymerization (ATRP); hyperbranched star copolymer; multi-responsive; controlled release
\end{abstract}

\footnotetext{
* E-mail: hongcy@ustc.edu.cn; pcy@ustc.edu.cn

Received May 5, 2012; published June 19, 2012.

Supporting information for this article is available free of charge via the Internet at http://sioc-journal.cn.

Project supported by the National Natural Science Foundation of China (Nos. 20974103, 21074121 and 21090354).

项目受国家自然科学基金(Nos. 20974103, 21074121, 21090354)资助.
} 


\section{1 引言}

两亲性聚合物形成的纳米粒子在药物可控释放以 及其它生物医药领域有着广泛的应用前景 ${ }^{[1 ~ 3]}$. 为了使 载药体系能准确到达目标位置, 并有效地控制药物的释 放, 以尽可能地减少副作用, 研究环境响应性的可控药 物释放体系是十分重要的内容. 目前已报道的不同环境 响应性的载药体系, 主要有氧化还原 ${ }^{[4,5]}$ 、温度 ${ }^{[6]} 、 \mathrm{pH}^{[3]}$ 、 磁场响应性 ${ }^{[7]}$ 等. 在温敏性的聚合物中, 聚甲基丙烯酸 2-(2-甲氧基乙氧基)乙酯( $\left.\mathrm{PMEO}_{2} \mathrm{MA}\right)$ 因其良好的生物相 容性和温敏性而备受关注 ${ }^{[8 \sim 10]}$, 它的低临界溶液温度 (LCST) 是 $26{ }^{\circ} \mathrm{C}^{[11]}$. Lutz 等 ${ }^{[12]}$ 曾报道用 $\mathrm{MEO}_{2} \mathrm{MA}$ 和甲基 丙烯酸寡聚乙二醇酯(OEGMA)进行无规共聚, 改变两 者的比例, 可以调节聚合物的 LCST 在 28 90 ${ }^{\circ} \mathrm{C}$ 之间. Davis 等 ${ }^{[13]}$ 曾报道将 $\mathrm{MEO}_{2} \mathrm{MA}$ 、OEGMA 和二甲基丙烯 酸乙二醇酯进行可逆加成一断裂一转移(RAFT)共聚反应 制备超支化聚合物, 改变配比可调节最终产物的 LCST, 并发现超支化聚合物的 LCST 比相应线形聚合物明显要 低. 作为载药体系材料应考虑的另一个重要因素是可降 解性. 二硫键因其对某些生物试剂(如谷胱甘肽)和化合 物(如二硫苏糖醇)非常敏感, 被广泛应用于合成环境响 应性和降解性的生物高分子材料 ${ }^{[14 ~ 18]}$. 其中绝大部分 使用含二硫键的引发剂, 或双(甲基)丙酰基的单体经可 控聚合制成线形聚合物、微凝胶或超支化聚合物 ${ }^{[14]}$. 只 有个别文献报道用含二硫键的自引发单体(inimer)进行 超支化(共)聚合反应, 生成可降解的超支化聚合物, 但 不具有环境响应特性 ${ }^{[19]}$.

相比线形聚合物，超支化聚合物有较大的内孔，可 以容纳客体分子, 因此具有多种潜在的应用, 例如控制 药物释放. 我们曾研究过用温敏性高分子修饰树状分子
的表面, 形成核/壳结构, 改变温度能有效地控制药物释 放 ${ }^{[20]}$. 与这一研究工作不同，本文合成了既有温敏性又 可降解的超支化聚合物, 再用 $\mathrm{pH}$ 响应性的高分子修饰 表面，形成核/壳结构，以期有效地控制药物的释放. 采 用的方法是首先合成一种含有二硫键的自引发单体，与 $\mathrm{MEO}_{2} \mathrm{MA}$ 进行自缩合乙烯基原子转移自由基共聚合 (SCVATRP), 得到的产物作大分子引发剂, 引发甲基丙 烯酸二甲氨基乙酯(DMAEMA)进行表面原子转移自由 基聚合(ATRP), 制得核/壳结构的星形聚合物. 以尼罗 红作为客体分子, 进行释放研究, 成功实现了 $\mathrm{pH}$ 和氧 化还原响应性的控制释放.

\section{2 结果与讨论}

为合成既有温敏性又能降解的超支化聚合物, 我们 没有采用文献上普遍使用的与含二硫键的双烯单体共 聚的方法，而是采用自缩合乙烯基聚合(SCVP), 因为后 者不仅分子量可控, 而且不会凝胶 ${ }^{[21]}$. 所用的自引发单 体为 BiBOE-S - MAOE. 与文献[19]所采用的合成方法 不同的是，通过双(2-羊全乙基)二硫化物与甲基丙烯酰氯 反应制备一端为羟基，另一端为双键的中间体. 为尽量 减少二硫化物的两端羟基都被酯化，我们将溶解在二氯 甲烷中的甲基丙烯酰氯稀溶液缓慢滴加到反应体系中. 即使如此, 产物中仍不可避免含双烯单体, 需经二氧化 硅柱色谱分离. 得到纯中间产物再与 2-溴代异丁酰溴反 应, 得到产物 BiBOE-S 2 -MAOE(见图式 1).

\section{1 超支化星形聚合物 H-PMEO ${ }_{2}$ MA-star-PDMAEMA 的合成}

我们利用 $\mathrm{BiBOE}-\mathrm{S}_{2}-\mathrm{MAOE}$ 和 $\mathrm{MEO}_{2} \mathrm{MA}$ (物质的量 比为 1/15)进行 ATRP 共聚合成超支化 $\mathrm{PMEO}_{2} \mathrm{MA}$. 图

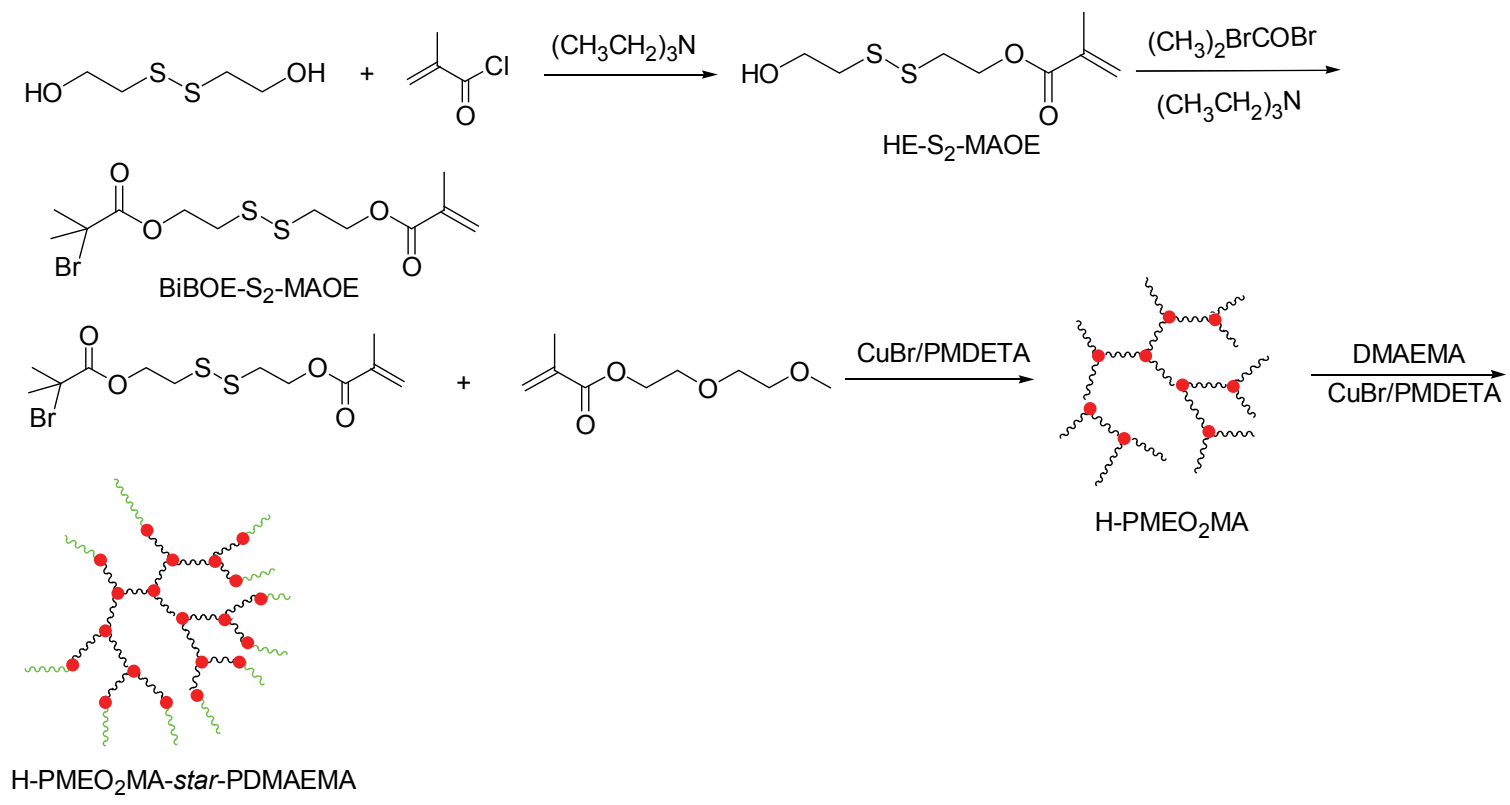

图式 1 H-PMEO ${ }_{2}$ MA-star-PDMAEMA 的合成

Scheme 1 Synthesis of H-PMEO ${ }_{2}$ MA-star-PDMAEMA 
$1 \mathrm{~B}$ 是超支化 $\mathrm{PMEO}_{2} \mathrm{MA}\left(\mathrm{HPMEO}_{2} \mathrm{MA}\right)$ 的 ${ }^{1} \mathrm{H}$ NMR 图. 质子峰的归属均标记在图上. 与 inimer $\mathrm{BiBOE}-\mathrm{S}_{2-}$ MAOE 的核磁共振氢谱图 $1 \mathrm{~A}$ 比较, 可以在图 $1 \mathrm{~B}$ 上清 楚地看到 $\delta 2.78$ 处的峰 $\mathrm{h}$, 这归属于紧靠 $\mathrm{S}$ 的亚甲基氢 $\left(\mathrm{CH}_{2} \mathrm{~S}_{2} \mathrm{CH}_{2}\right)$. 而结构单元 $\mathrm{MEO}_{2} \mathrm{MA}$ 上侧基的醚甲基氢 的吸收峰出现在 $\delta 3.4$ 处(e), 这说明 BiBOE-S - -MAOE 与 $\mathrm{MEO}_{2} \mathrm{MA}$ 发生了共聚. 通过比较峰 $\mathrm{e}$ 和峰 $\mathrm{h}$ 的面积, 可以计算出超支化聚合物 $\mathrm{HPMEO}_{2} \mathrm{MA}$ 中 $\mathrm{MEO}_{2} \mathrm{MA}$ 与 BiBOE-S ${ }_{2}-\mathrm{MAOE}$ 结构单元的比值为 11.5/1. 图 2(a)是 $\mathrm{HPMEO}_{2} \mathrm{MA}$ 的 GPC 谱图. 数均分子量 $\left(M_{\mathrm{n}}\right)$ 和分子量分 布 $\left(M_{\mathrm{w}} / M_{\mathrm{n}}\right)$ 分别为 $8300 \mathrm{~g} / \mathrm{mol}$ 和 2.61. 多峰和宽分子量 分布是超支化聚合物的一个特征.

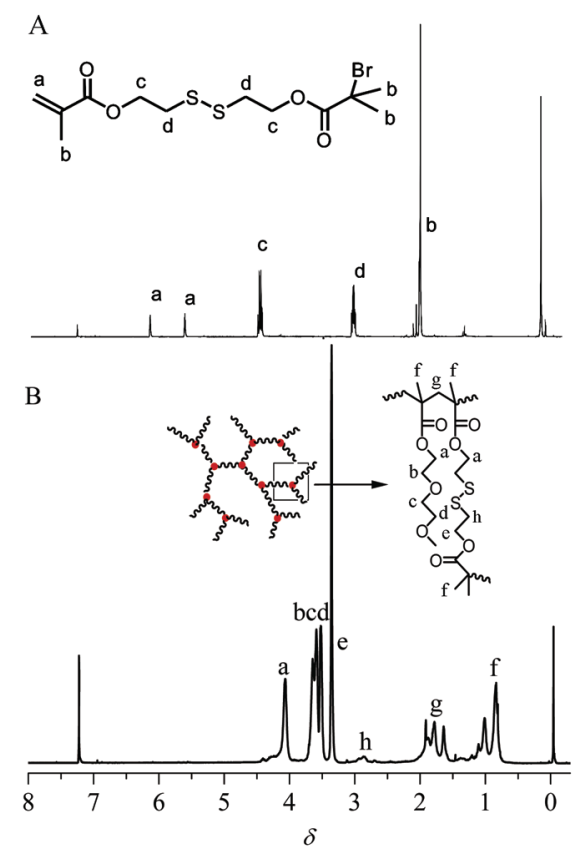

图 1 BiBOE- $\mathrm{S}_{2}-\mathrm{MAOE}(\mathrm{A})$ 和超支化 $\mathrm{PMEO}_{2} \mathrm{MA}(\mathrm{B})$ 的 ${ }^{1} \mathrm{H}$ NMR 谱图 $\left(\mathrm{CDCl}_{3}\right)$

Figure $1{ }^{1} \mathrm{H}$ NMR spectra of BiBOE- $\mathrm{S}_{2}-\mathrm{MAOE}(\mathrm{A})$ and hyperbranched $\mathrm{PMEO}_{2} \mathrm{MA}(\mathrm{B})$ in $\mathrm{CDCl}_{3}$

$\mathrm{HPMEO}_{2} \mathrm{MA}$ 作为大分子引发剂, 引发 DMAEMA 的 ATRP 反应, 分别聚合 30,60 和 $90 \mathrm{~min}$, 得到了不同 分子量的超支化星形聚合物, 分别标记为 $\mathrm{H}-\mathrm{PMEO}_{2} \mathrm{MA}-$ star-PDMAEMA-30, 60, 90. 图 2b, 2c 和 $2 \mathrm{~d}$ 分别对应上 述聚合时间所得的聚合物的 GPC 谱图. 可以看到, 随着
反应时间的增加, GPC 图向分子量大的方向移动. 反应 时间越长，分子量越大，而且分子量分布也越来越窄. 这是因为 ATRP 是可控聚合反应，所得 PDMAEMA 的 分子量分布比较窄，随该聚合物分子量增加，多分散性 的超支化聚合物核对星形聚合物分子量分布的影响越 来越小.

为了表征星形聚合物的结构和组成, 我们测定了 H-PMEO ${ }_{2}$ MA-star-PDMAEMA 的核磁共振氢谱, H-PMEO ${ }_{2}$ MA-star-PDMAEMA-60 的结果如图 3 所示. 各质子峰的归属标注在图上. 与图 $1 \mathrm{~B}$ 相比, 在 $\delta 2.3$ (b) 和 $\delta 2.6$ (c) 出现了 PDMAEMA 的特征峰, 分别为与叔氮 相邻的甲基和亚甲基的质子峰. 而 $\mathrm{MEO}_{2} \mathrm{MA}$ 单元上醚 甲基质子峰出现在 $\delta 3.4$ (a)处，这说明 PDMAEMA 接枝 到 $\mathrm{H}-\mathrm{PMEO}_{2} \mathrm{MA}$ 表面. 根据这两质子峰的积分面积比, 即 $b / a$, 可以算出聚合物中 DMAEMA 单元和 $M^{2} O_{2} M A$ 单元的比例, 计算结果列于表 1. 结果显示随聚合反应 时间增加, 星形聚合物中 DMAEMA 的含量增加, 即该 比值增加.

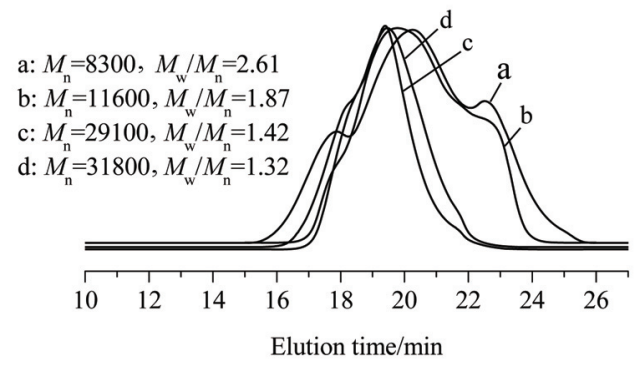

图 2 GPC 图, (a) 是 $\mathrm{H}-\mathrm{PMEO}_{2} \mathrm{MA}$, (b), (c), (d) 分别是 $\mathrm{H}-\mathrm{PMEO}_{2} \mathrm{MA}$ 引发 DMAEMA 聚合 $30,60,90$ min 得到的产物

Figure 2 GPC curves of $\mathrm{H}-\mathrm{PMEO}_{2} \mathrm{MA}$ and $\mathrm{H}-\mathrm{PMEO}_{2} \mathrm{MA}$-starPDMAEMAs obtained from ATRP of DMAEMA for 30, 60 and $90 \mathrm{~min}$, respectively

\section{$2.2 \mathrm{H}-\mathrm{PMEO}_{2} \mathrm{MA}$ 和 $\mathrm{H}-\mathrm{PMEO}_{2} \mathrm{MA}$-star-PDMAEMA 的温敏性研究}

Davis 等 ${ }^{[13]}$ 曾报道高分子的支化结构会影响聚合物 的温敏性能，相对于线形聚合物，超支化聚合物的 LCST 会有所降低. 在我们的研究工作中也观察到了这 一现象. 通常, 线形 $\mathrm{PMEO}_{2} \mathrm{MA}$ 的 LCST 在室温附近 ${ }^{[22]}$. 我们制得的 H-PMEO ${ }_{2} \mathrm{MA}$ 水溶液 $(4 \mathrm{mg} / \mathrm{mL})$ 的 LCST 在 $10{ }^{\circ} \mathrm{C}$ 以下, 超出仪器的测量范围. 但在 $2{ }^{\circ} \mathrm{C}$ 放置一会

表 1 超支化星形聚合物 H-PMEO ${ }_{2}$ MA-star-PDMAEMA 的合成

Table 1 Synthesis of H-PMEO 2 MA-star-PDMAEMA

\begin{tabular}{lllll}
\hline Sample $^{a}$ & Time $/ \mathrm{min}$ & $M_{\mathrm{n}}{ }^{b} /\left(\mathrm{g} \cdot \mathrm{mol}^{-1}\right)$ & $M_{\mathrm{w}} / M_{\mathrm{n}}{ }^{b}$ & $R^{c}$ \\
\hline $\mathrm{H}_{-} \mathrm{PMEO}_{2} \mathrm{MA}$ & 0 & 8300 & 2.61 & 0 \\
$\mathrm{H}_{-} \mathrm{PMEO}_{2}$ MA-star-PDMAEMA-30 & 30 & 11600 & 1.87 & 0.6 \\
$\mathrm{H}_{-} \mathrm{PMEO}_{2}$ MA-star-PDMAEMA-60 & 60 & 29100 & 1.42 & 1.3 \\
$\mathrm{H}_{-} \mathrm{PMEO}_{2} \mathrm{MA}$-star-PDMAEMA-90 & 90 & 31800 & 1.32 & 1.7 \\
\hline
\end{tabular}

${ }^{a} \mathrm{H}_{-\mathrm{PMEO}_{2} \mathrm{MA}}$ was prepared by ATRP, BiBOE-S $2-\mathrm{MAOE} / \mathrm{MEO}_{2} \mathrm{MA} / \mathrm{CuBr} / \mathrm{PMDETA}=10 / 152 / 1 / 2$ (molar ratio), $50{ }^{\circ} \mathrm{C}, 48 \mathrm{~h}$; H-PMEO ${ }_{2} \mathrm{MA}_{\text {star-PDMAEMAs }}$

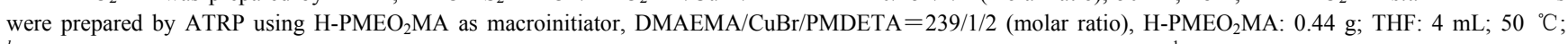

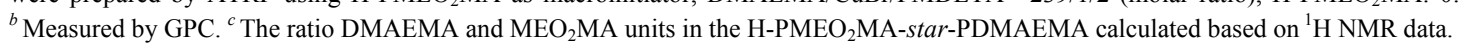




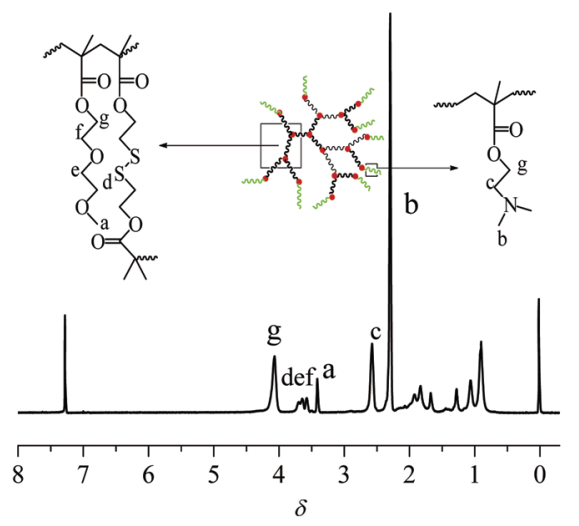

图 3 超支化星形聚合物 $\mathrm{H}-\mathrm{PMEO}_{2} \mathrm{MA}$-star-PDMAEMA-60 的 ${ }^{1} \mathrm{H}$ $\mathrm{NMR}$ 谱图 $\left(\mathrm{CDCl}_{3}\right)$

Figure $3 \quad{ }^{1} \mathrm{H}$ NMR spectrum of hyperbranched star copolymer, H-PMEO ${ }_{2}$ MA-star-PDMAEMA-60 in $\mathrm{CDCl}_{3}$

后, 可得到完全透明的聚合物溶液. 所以我们推测该聚 合物的 LCST 在 2 10 ${ }^{\circ} \mathrm{C}$ 之间. 即在室温条件下, 该聚 合物溶液处于 LCST 以上，呈现非水溶性.

颜德岳等 ${ }^{[23]}$ 曾报道 PEO 的链长对超支化星形聚合 物(HBPO-star-PEO)的水溶性有显著的影响, 改变 PEO 链的长度, 星形聚合物的 LCST 可以在 $8 \sim 81{ }^{\circ} \mathrm{C}$ 进行调
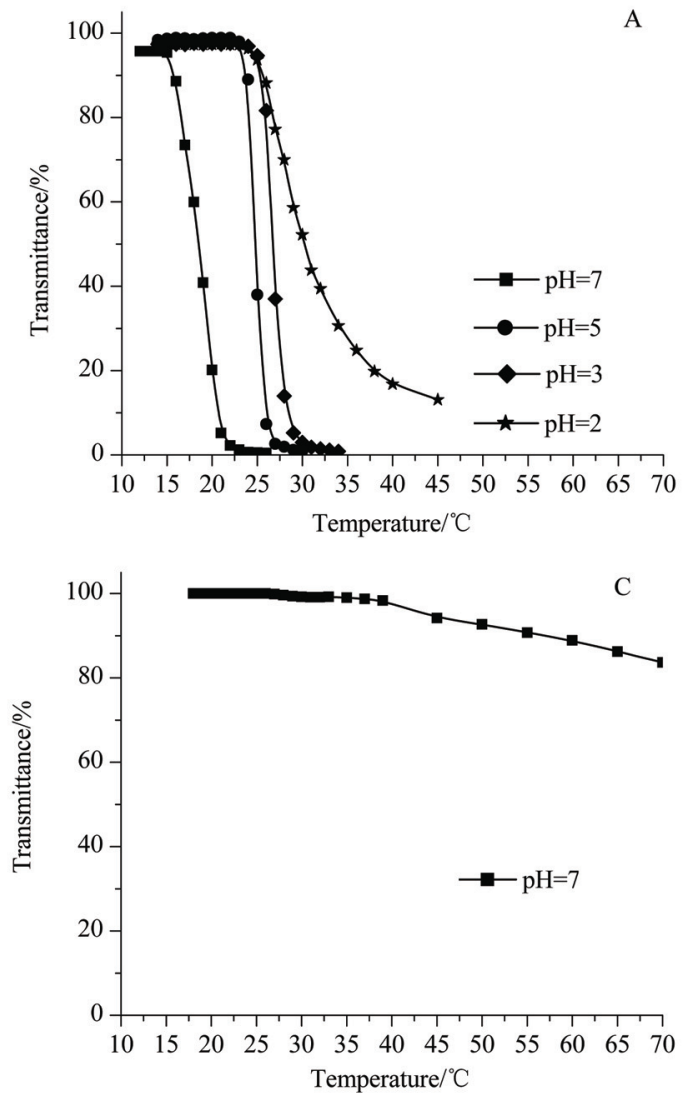

节. 我们利用 $\mathrm{H}-\mathrm{PMEO}_{2} \mathrm{MA}$ 作大分子引发剂, 引发 DMAEMA 进行 ATRP, 合成具有不同 PDMAEMA 链长 的 H-PMEO ${ }_{2}$ MA-star-PDMAEMA, 研究其溶液行为, 结 果列于表 2 .

表 2 的结果显示, 在 $\mathrm{pH}=7$ 时, H-PMEO ${ }_{2}$ MA-starPDMAEMA-30 和 H-PMEO ${ }_{2}$ MA-star-PDMAEMA-60 的 LCST 分别为 17 和 $23{ }^{\circ} \mathrm{C}$. 当接枝更长的 PDMAEMA 链即为 H-PMEO $\mathrm{O}_{2}$ MA-star-PDMAEMA-90 时, 随着温度 升高, 透过率降低缓慢且变化很小, 几乎很难界定 LCST(见图 4C 和 4D). 这说明随着外层 PDMAEMA 链 段长度的增加, 稳定收缩的超支化聚合物核的能力越 强, LCST 有所提高, 甚至聚集形成较为稳定粒子.

PDMAEMA 具有 $\mathrm{pH}$ 敏感性, 在酸性条件下, 聚合 物链伸展, 水溶性增加. 在碱性溶液中, 聚合物链收缩, 亲水性降低, 稳定聚合物粒子的能力下降. 因此, 溶液 酸碱度对星形聚合物的 LCST 有较大影响. 无论是 H-PMEO ${ }_{2}$ MA-star-PDMAEMA-30 还是 H-PMEO ${ }_{2}$ MAstar-PDMAEMA-60，随溶液的酸度增加，即 $\mathrm{pH}$ 值降低, LCST 有所升高, 尤其是当 $\mathrm{pH}$ 从 7 变到 5 时, 两者的 LCST 都有明显升高. 当溶液 $\mathrm{pH}$ 值在 5 以下，聚合物的 LCST 随 $\mathrm{pH}$ 变化不明显(见表 2). 当溶液从 $\mathrm{pH}=5$ 降低
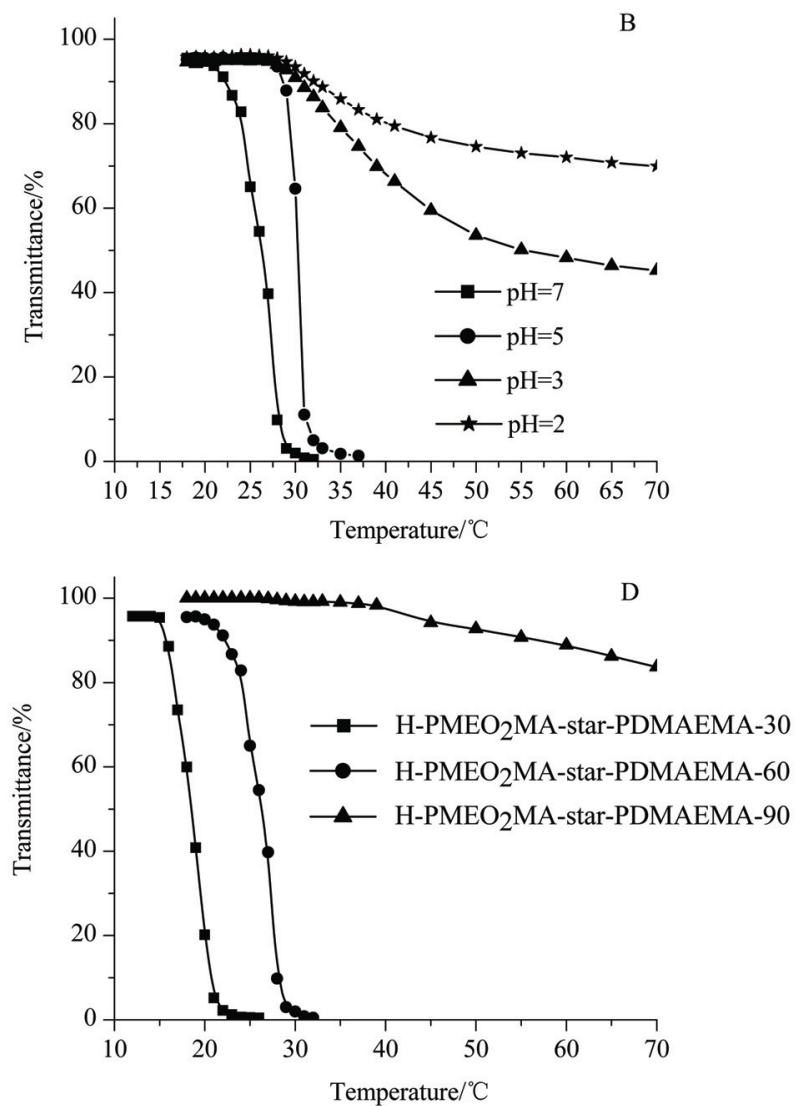

图 4 H-PMEO ${ }_{2}$ MA-star-PDMAEMA-30 (A), H-PMEO ${ }_{2}$ MA-star-PDMAEMA-60 (B) 和 H-PMEO 2 MA-star-PDMAEMA-90 (C)的不同 pH 值水溶液和 $\mathrm{pH}=7$ 的水溶液(D)的透射率随温度的变化曲线. 聚合物浓度: $4 \mathrm{mg} / \mathrm{mL}, \lambda=500 \mathrm{~nm}$

Figure 4 Temperature dependence of the optical transmittance of the H-PMEO ${ }_{2}$ MA-star-PDMAEMA-30 (A), H-PMEO ${ }_{2}$ MA-star-PDMAEMA-60 (B) and $\mathrm{H}-\mathrm{PMEO}_{2}$ MA-star-PDMAEMA-90 (C) aqueous solutions at various $\mathrm{pHs}$, and at $\mathrm{pH}=7$ (D). Concentration: $4 \mathrm{mg} / \mathrm{mL} ; \lambda=500 \mathrm{~nm}$. 
表 2 在不同 $\mathrm{pH}$ 下, $\mathrm{H}-\mathrm{PMEO}_{2}$ MA-star-PDMAEMA 的低临界溶液温度(LCST)

Table 2 The LCST of H-PMEO ${ }_{2}$ MA-star-PDMAEMA at different $\mathrm{pH}$

\begin{tabular}{|c|c|c|c|c|}
\hline \multirow{2}{*}{ Sample $^{a}$} & \multicolumn{4}{|c|}{$\mathrm{LCST} /{ }^{\circ} \mathrm{C}$} \\
\hline & $\mathrm{pH}=7$ & $\mathrm{pH}=5$ & $\mathrm{pH}=3$ & $\mathrm{pH}=2$ \\
\hline H-PMEO ${ }_{2}$ MA-star-PDMAEMA-30 & 17 & 24 & 26 & 26 \\
\hline H-PMEO ${ }_{2}$ MA-star-PDMAEMA-60 & 23 & 29 & 31 & 32 \\
\hline H-PMEO ${ }_{2}$ MA-star-PDMAEMA-90 & $-^{b}$ & $-^{b}$ & $-^{b}$ & $-^{b}$ \\
\hline
\end{tabular}

${ }^{a}$ The same as in Table $1 ;^{b}$ - refers that the dependence of temperature-transmittance was too small to be detected accurately.

到 $\mathrm{pH}=3$ 时, 图 $4 \mathrm{~A}$ 和 $4 \mathrm{~B}$ 上的温度-透过率曲线有很大 的不同, 即在 $\mathrm{pH}=3$ 时, 对 H-PMEO ${ }_{2} \mathrm{MA}-$ starPDMAEMA-30, 该曲线仍存在突变; 而 H-PMEO ${ }_{2} \mathrm{MA}-$ star-PDMAEMA-60 的曲线变化平缓, 且在 $60{ }^{\circ} \mathrm{C}$ 以后, 透过率始终维持在 $50 \%$ 左右. 这进一步说明对不同 PDMAEMA 链长的星形聚合物的 LCST 行为的影响是 不同的. 图 4C 显示在 $\mathrm{pH}=7$ 时, H-PMEO ${ }_{2} \mathrm{MA}-$ starPDMAEMA-90 溶液的透过率随温度升高很难降低到 $80 \%$ 以下, $\mathrm{pH}$ 低于 7 时, 温度-透过率曲线变化更不明 显. 以上结果均说明随着 $\mathrm{pH}$ 降低, PDMAEMA 的质子 化程度不断提高, 链段之间的排斥力增强, 使得 $\mathrm{H}-\mathrm{PMEO}_{2} \mathrm{MA}$-star-PDMAEMA 分子间的聚集变得越来 越困难, 这与以前报道的结果是一致的 ${ }^{[24,25]}$. 另外, PDMAEMA 也具有温敏性, 但是其 LCST 受 $\mathrm{pH}$ 影响较 大, 在 $\mathrm{pH}<8.2$ 时甚至观测不到 $\mathrm{LCST}^{[26]}$. 所以本文中 PDMAEMA 臂的温敏 性 对 H-PMEO 2 MA-starPDMAEMA 是没有影响的.

\section{3 纳米粒子的制备、表征以及控制释放研究}

为了证明超支化星形聚合物在 LCST 以上形成了胶 束, 我们对聚合物样品用 DLS 和 TEM 进行表征. 以 H-PMEO ${ }_{2}$ MA-star-PDMAEMA-60 为例, 在 $\mathrm{pH}=5$ 和 7 的水溶液中, $25{ }^{\circ} \mathrm{C}$ 分别处在它的 LCST 以下和以上(图 $4 \mathrm{~B}$ ). 图 5 是这两种情况下的 TEM 照片, $\mathrm{pH}=5$ 时, 得到 了粒径 20 30 nm 且分布比较均匀的胶束(图 5A), 说明 H-PMEO 2 MA-star-PDMAEMA-60 不是以单分子的形式 存在, 而是组装成了小胶束. 可能是因为超支化星形聚 合物内层的 $\mathrm{H}-\mathrm{PMEO}_{2} \mathrm{MA}$ 是疏水的, 被水溶性的 PDMAEMA 稳定. DLS 测得粒径(41.2 nm) 比 TEM 测得 的结果偏大, 可能是因为 DLS 是在溶液中测得, 此时胶 束壳层的分子处于伸展状态, 而 TEM 是在干态时测得, 此时胶束壳层处于塌缩状态. 而在 $\mathrm{pH}=7$ 的水溶液中, $25{ }^{\circ} \mathrm{C}$ 已超过 H-PMEO ${ }_{2}$ MA-star-PDMAEMA-60 的 LCST, PDMAEMA 已稳定不了疏水胶束, 纳米粒子便 无规地聚集在一起, 形成不规则的形貌(图 5B), DLS 测 得其平均粒径为 $417.0 \mathrm{~nm}$. 前面推测, 超支化聚合物 $\mathrm{H}-\mathrm{PMEO}_{2} \mathrm{MA}$ 的 LCST 在 $2 \sim 10{ }^{\circ} \mathrm{C}$ 之间, 所以在 $2{ }^{\circ} \mathrm{C}$ 下, 将 H-PMEO ${ }_{2}$ MA-star-PDMAEMA-60 溶解在含有客 体分子的水溶液中, 将温度升高至室温, 就可将客体分 子包裹在聚合物胶束内. 本研究就是采用这种方法将尼 罗红包裹在 H-PMEO 2 MA-star-PDMAEMA-60 胶束内
的. 选择尼罗红作为客体分子, 是因为它以分子形式溶 解在水溶液中, 在 $630 \mathrm{~nm}$ 处的荧光可以忽略不计; 而在 疏水的胶束中, 其荧光强度将显著增加 ${ }^{[27,28]}$. 这样未包 裹的尼罗红对下一步的释放研究影响较小. 图 6 是在不 同条件下，包裹在 H-PMEO ${ }_{2}$ MA-star-PDMAEMA-60 胶 束中的尼罗红的荧光强度随时间的变化. 图 $6 \mathrm{~A} \sim 6 \mathrm{C}$ 测 定不同时间溶液的苂光光谱的结果显示, 溶液的 $\mathrm{pH}$ 值 对尼罗红的释放行为影响不大, 只是随酸性增加, 即从 $\mathrm{pH}=7$ 减少到 $\mathrm{pH}=5$, 再到 3 , 释放的尼罗红稍微增加. 因为在核/壳星形聚合物中, 仅壳层 PDMAEMA 是酸敏 的. 溶胀在壳层的尼罗红随 $\mathrm{pH}$ 减小释放量增加. 相对 于壳层, 绝大部分尼罗红包埋在核中, 而这部分客体分 子几乎不释放. 为了证实尼罗红包埋在核中, 并能可控 释放, 将这一释药体系放置在二硫苏糖醇(DTT)的水溶 液 $(0.2 \mathrm{mg} / \mathrm{mL})$ 中, 测定不同时间溶液的荧光光谱, 结果 如图 6D 所示. 可以看到随时间增加, 包埋在超支化聚 合物核内的客体分子逐渐释放. 这是因为超支化聚合物 $\mathrm{H}-\mathrm{PMEO}_{2} \mathrm{MA}$ 内引入了二硫键，在生物试剂如谷胱甘肽 和化合物如 DTT 和三丁基膦等存在下, $\mathrm{S}-\mathrm{S}$ 键断 裂 ${ }^{[16,17]}$, 使超支化结构逐渐解体, 从而释放出包裹在里 面的尼罗红.
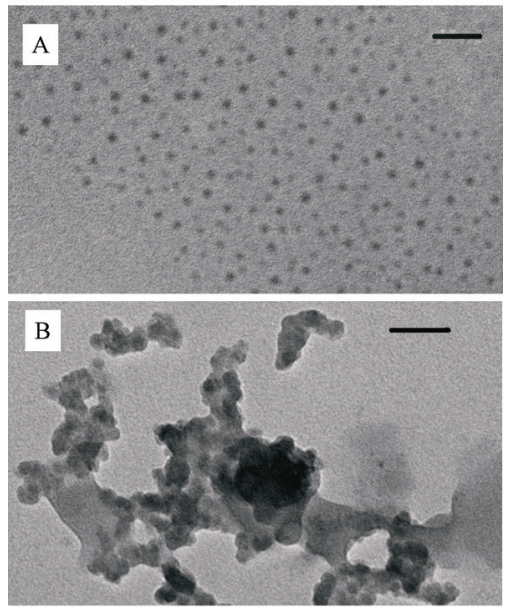

图 5 分别从 $\mathrm{pH}=5$ (A) 和 $\mathrm{pH}=7$ (B) 的 $\mathrm{H}-\mathrm{PMEO}_{2} \mathrm{MA}$-starPDMAEMA-60 的水溶液所制得样品的 TEM 图片. 标尺: (A) $100 \mathrm{~nm}$, (B) $200 \mathrm{~nm}$

Figure 5 TEM images of H-PMEO ${ }_{2}$ MA-star-PDMAEMA-60 samples prepared from its aqueous solutions at $\mathrm{pH}=5$ (A) and $\mathrm{pH}=7$ (B). Scale bars: (A) $100 \mathrm{~nm}$, (B) $200 \mathrm{~nm}$ 

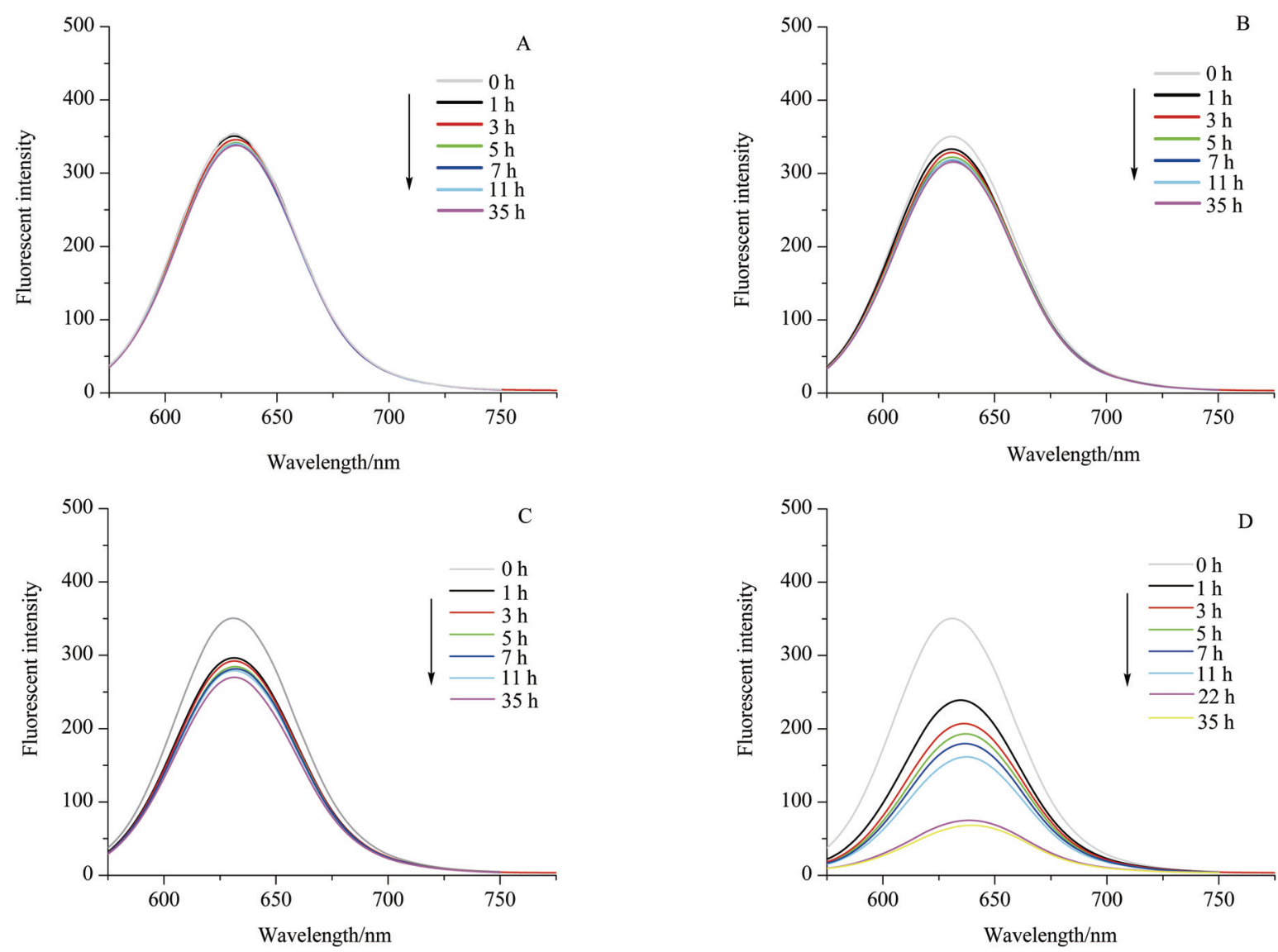

图 6 H-PMEO ${ }_{2}$ MA-star-PDMAEMA-60-尼罗红释药系统在不同 $\mathrm{pH}$ 值或含 DTT 的水溶液中的释放研究, (A) $\mathrm{pH}=7,(\mathrm{~B}) \mathrm{pH}=5,(\mathrm{C}) \mathrm{pH}=3,(\mathrm{D})$ DTT. 聚合物浓度: $3.75 \mathrm{mg} / \mathrm{mL}$; 温度: $25{ }^{\circ} \mathrm{C} ; \lambda=630 \mathrm{~nm}$

Figure 6 The Nile Red release for the H-PMEO 2 MA-star-PDMAEMA-60 micelles containing Nile Red at different aqueous solution, (A) $\mathrm{pH}=7$, (B) $\mathrm{pH}$ $=5$, (C) $\mathrm{pH}=3$, (D) DTT. Concentration: $3.75 \mathrm{mg} / \mathrm{mL}$; Temperature: $25{ }^{\circ} \mathrm{C} ; \lambda=630 \mathrm{~nm}$

\section{3 结论}

采用 ATRP 和 SCVP 相结合的方法成功地合成了超 支化星形聚合物 H-PMEO 2 MA-star-PDMAEMA。该聚 合物具有温度、 $\mathrm{pH}$ 以及氧化还原多重响应性. H-PMEO ${ }_{2} \mathrm{MA}$ 的 LCST 在 $2 \sim 10{ }^{\circ} \mathrm{C}$. 共聚物组成、链长 和溶液的 $\mathrm{pH}$ 影响共聚物的 LCST. 随 PDMAEMA 链长 增加, LCST 升高; 当 DMAEMA/MEO $\mathrm{MEA}_{2} \mathrm{MA}$. 时, 温 度-透射率曲线变化很小, LCST 难以精确测定. 溶液的 $\mathrm{pH}$ 降低, LCST 升高. 在室温下, 星形聚合物能形成 $\mathrm{H}-\mathrm{PMEO}_{2} \mathrm{MA}$ 为核, PDMAEMA 为壳的胶束. 尼罗红H-PMEO ${ }_{2}$ MA-star-PDMAEMA 载药体系释放实验的结 果表明, 该载药体系具有 $\mathrm{pH}$ 和氧化还原响应性释放的 特性，在生物医药领域有着潜在的应用前景.

\section{4 实验部分}

2- 羟乙基-2'- 甲基丙烯酸乙酯-双硫化物 (HE-S 2 -MAOE)和 2-(2'-溴代异丁酰氧)乙基-甲基丙烯酰 氧一乙基-双硫化物(BiBOE-S ${ }_{2}$-MAOE) 的合成见支持信 息.

\section{1 超支化聚合物 $\mathrm{H}-\mathrm{PMEO}_{2} \mathrm{MA}$ 的合成}

将 BiBOE-S 2 -MAOE ( $0.30 \mathrm{~g}, 0.81 \mathrm{mmol}), \mathrm{MEO}_{2} \mathrm{MA}$ (2.29 g, $12.15 \mathrm{mmol}), \mathrm{CuBr}(12 \mathrm{mg}, 0.08 \mathrm{mmol}$ )和配体 PMDETA $(30 \mathrm{mg} 0.16 \mathrm{mmol}$ )以及四氢呋喃 $(3 \mathrm{~mL})$ 加入 到一个 $5 \mathrm{~mL}$ 的反应管中. 经冷冻-抽真空-解冻三次循 环，除去反应液中的氧气，封管. 在油浴温度 $50{ }^{\circ} \mathrm{C}$ 下 反应 $48 \mathrm{~h}$. 加入 $20 \mathrm{~mL}$ 四氢呋喃稀释后, 过中性氧化铝 柱除去铜盐. 溶液浓缩后在石油醚中反复沉淀三次, 真 空干燥至恒重, 得到产物 $2.09 \mathrm{~g}$.

\section{2 超支化星形聚合物 (H-PMEO 2 MA-star- PDMAEMA)的合成}

将 $\mathrm{H}-\mathrm{PMEO}_{2} \mathrm{MA}(0.44 \mathrm{~g})$, DMAEMA (3.00 g, 19.1 $\mathrm{mmol}), \mathrm{CuBr}(12 \mathrm{mg}, 0.08 \mathrm{mmol}$ )和配体 PMDETA (30 $\mathrm{mg} 0.16 \mathrm{mmol})$ 以及四氢呋喃 $(4 \mathrm{~mL})$ 加入到 $10 \mathrm{~mL}$ 的反 应管中. 经三次冷冻-抽真空-解冻循环，除去反应液中 的氧气, 封管, 在 $50{ }^{\circ} \mathrm{C}$ 下应 $0.5 \mathrm{~h}$. 加 $20 \mathrm{~mL}$ 四氢呋 喃稀释后, 过中性氧化铝柱子除去铜盐. 溶液浓缩后在 石油醚中反复沉淀三次, 真空干燥至恒重, 得到产物 $0.57 \mathrm{~g}$. 


\section{3 低临界溶液温度(LCST)的测试}

取 H-PMEO ${ }_{2}$ MA-star-PDMAEMA $(20 \mathrm{mg})$ 加入到 10 $\mathrm{mL}$ 的玻璃瓶中, 再加入 $5 \mathrm{~mL}$ 蒸馏水, 超声分散. 因聚 合物的 LCST 低于室温, 所以分散液在冰箱中 $\left(2{ }^{\circ} \mathrm{C}\right)$ 放 置过夜以保证聚合物充分溶解. 在 $\lambda=500 \mathrm{~nm}$ 的光照下, 测定聚合物溶液在不同温度下的透过率. 每个温度平衡 $10 \mathrm{~min}$ 再测定, 以透过率降低为 $90 \%$ 的温度作为该聚合 物溶液的 LCST.

\section{4 客体分子的装载与释放研究}

以尼罗红作为模型药物, 将该客体分子的甲醇溶液 $\left(1.2 \mathrm{~mL}, 5 \times 10^{-4} \mathrm{~mol} / \mathrm{L}\right)$ 加入到 $18 \mathrm{~mL}$ 的聚合物水溶液(4 $\left.\mathrm{mg} / \mathrm{mL}, 2{ }^{\circ} \mathrm{C}\right)$ 中. 在 $25{ }^{\circ} \mathrm{C}$ 下搅拌过夜, 逐渐形成包裹 尼罗红的胶束溶液. 将所得到的样品平均分成四份, 分 别加入 $0.5 \mathrm{~mL}$ DTT 水溶液, $0.5 \mathrm{~mL}$ 蒸馏水, $0.5 \mathrm{~mL} \mathrm{pH}=$ 2 的水溶液, $0.5 \mathrm{~mL} \mathrm{pH}=4$ 的水溶液. 在不同时间间隔 测定样品的荧光光谱.

\section{References}

[1] Sun, T. M.; Du, J. Z.; Yan, L. F.; Mao, H. Q.; Wang, J. Biomaterials 2008, 29, 4348.

[2] Huang, X. W.; Gu, L. N.; Lu, G. L.; Huang, X.; Zhang, Y. Q.; Huang, X. Y. Acta Chim. Sinica 2009, 67, 1363. (黄晓炜, 顾丽娜, 陆国林, 黄啸, 张亚琴, 黄晓宇, 化学学报, 2009, 67, 1363.)

[3] Zhu, Y. M.; Zhang, Y.; Liu, Z. L.; Lang, M. D. Acta Chim. Sinica 2010, 68, 2449. (朱亚明, 张琰, 刘子路, 郎美东, 化学学报, 2010, $68,2449$.

[4] Lv, L. P.; Xu, J. P.; Liu, X. S.; Liu, G. Y.; Yang, X. A.; Ji, J. A. Macromol. Chem. Phys. 2010, 211, 2292.

[5] Sun, J. T.; Hong, C. Y.; Pan, C. Y. J. Phys. Chem. C 2010, 114, 12481.

[6] Morishima, Y. Angew. Chem. Int. Ed. 2007, 46, 1370.
[7] Pan, D. K.; Zhang, H. Acta Chim. Sinica 2011, 69, 1545. (盘登科, 张慧, 化学学报, 2011, 69, 1545.)

[8] Wang, Y.; Li, X.; Hong, C. Y.; Pan, C. Y. J. Polym. Sci. Part A: Polym. Chem. 2011, 49, 3280.

[9] York, A. W.; Kirkland, S. E.; McCormick, C. L. Adv. Drug Delivery Rev. 2008, 60, 1018.

[10] Boyer, C.; Bulmus, V.; Davis, T. P.; Ladmiral, V.; Liu, J. Q.; Perrier, S. Chem. Rev. 2009, 109, 5402.

[11] Han, S.; Hagiwara, M.; Ishizone, T. Macromolecules 2003, 36, 8312.

[12] Lutz, J. F.; Hoth, A. Macromolecules 2006, 39, 893.

[13] Luzon, M.; Boyer, C.; Peinado, C.; Corrales, T.; Whittaker, M.; Tao, L.; Davis, T. P. J. Polym. Sci. Part A: Polym. Chem. 2010, 48, 2783.

[14] Rikkou, M. D.; Patrickios, C. S. Prog. Polym. Sci. 2011, 36, 1079.

[15] Li, C. M.; Madsen, J.; Armes, S. P.; Lewis, A. L. Angew. Chem. Int Ed. 2006, 45, 3510.

[16] Hong, C. Y.; You, Y. Z.; Wu, D. C.; Liu, Y.; Pan, C. Y. J. Am. Chem. Soc. 2007, 129, 5354.

[17] Wang, L.; Li, C. M.; Ryan, A. J.; Armes, S. P. Adv. Mater. 2006, 18, 1566.

[18] Yang, W.; Pan, C. Y.; Liu, X. Q.; Wang, J. Biomacromolecules 2011, 12, 1523 .

[19] Tsarevsky, N. V.; Huang, J.; Matyjaszewski, K. J. Polym. Sci. Part A: Polym. Chem. 2009, 47, 6839.

[20] You, Y. Z.; Hong, C. Y.; Pan, C. Y.; Wang, W.-P. Adv. Mater. 2004, 16,1953

[21] Jia, Z. F.; Yan, D. Y. J. Polym. Sci. Part A: Polym. Chem. 2005, 43, 3502 .

[22] Paris, R.; Quijada-Garrido, I. Eur. Polym. J. 2009, 45, 3418.

[23] Zhou, Y. F.; Yan, D. Y.; Dong, W. Y.; Tian, Y. J. Phys. Chem. B 2007, 111, 1262.

[24] Baines, F. L.; Billingham, N. C.; Armes, S. P. Macromolecules 1996, 29, 3416.

[25] Xiao, G. L.; Hu, Z. B.; Zeng, G. P.; Wang, Y. Q.; Huang, Y. Q. Hong, X. L.; Xia, B. L.; Zhang, G. Y. J. Appl. Polym. Sci. 2012, $124,202$.

[26] Ren, F.; Chen, X. N.; Xia, Y. Z.; Shi, S. X.; Jiao, S. K. Acta Polym. Sin. 2007，(9)，838. (任芳，陈晓农，夏宇正，石淑先，焦书科，高 分子学报, 2007, (9), 838.)

[27] Gillies, E. R.; Jonsson, T. B.; Frechet, J. M. J. J. Am. Chem. Soc. 2004, 126, 11936.

[28] Jiang, X. G.; Zhao, B. Macromolecules 2008, 41, 9366. 